Trauma Berufskrankh 2015 • 17[Suppl 1]:241-243 DOI 10.1007/s10039-014-2126-y

Online publiziert: 22. Januar 2015

(c) Springer-Verlag Berlin Heidelberg 2015

\section{M. Öhlbauer}

Abteilung für Plastische Chirurgie und Verbrennungsmedizin, BG-Unfallklinik Murnau

\section{Thermische Verletzungen im Kindes- und Jugendalter}

re der Verletzung wird bei Kindern und Jugendlichen, wie bei Erwachsenen auch, in Prozent KOF sowie in der Tiefenausdehnung (Grad) bemessen. Begleitverletzungen, insbesondere das Inhalationstrauma, müssen bei der Beurteilung mitberücksichtigt werden.

Die Ausdehnung der Brandverletzung wird im Kindes- und Jugendalter nach dem Schema von Lund u. Browder beurteilt ( $\bullet$ Tab. 1). Zur ersten groben Einschätzung am Unfallort sowie auch bei der Erstaufnahme bewährte sich die Handflächenformel, bei der die Handfläche (mit den Fingern) des Patienten 1\% der Körperoberfläche des Brandverletzten entspricht.

Hinsichtlich der Tiefenausdehnung lassen sich Brandverletzungen in 3 Grade einteilen:
Grad 1, epidermal: Rötung, Schwellung, starker Schmerz, intaktes Epithel Grad 2a, oberflächlich dermal: Blasenbildung, feuchter hyperämischer Wundgrund, prompte Rekapillarisierung, Schmerz (• Abb. 1)

Grad 2b, tief dermal: fetzenförmige Epidermolyse, Blasenbildung, weißlicher, feuchter Wundgrund, gestörte Rekapillarisierung, Hautanhangsgebilde partiell vorhanden, Haarfollikel und Schweißdrüsenausführungsgänge mitbetroffen und teilweise zerstört, mäßiger Schmerz

\section{(- Abb. 2)}

Grad 3, komplett dermal: trockene, weiße, elfenbeinfarbene Hautnekrose bis hin zur Verkohlung, Verlust von Hautanhangsgebilden, keine Schmerzen

(- Abb. 3) Hautanhangsgebilde intakt, starker

- Verbrennungen von mehr als 10\% KOF (Körperoberfläche),

- Verbrennungen von Gesicht, Händen, Füßen, Anogenitalregion sowie

- Inhalationsverletzungen.

Beruhen bei Kindern über 70\% der thermischen Verletzungen zumeist auf Schädigungen durch Verbrühung, treten im Jugendalter Flammenverbrennungen und Stromverletzungen in den Vordergrund.

\section{Einstufung der Verletzungsschwere}

Thermische oder chemische Einwirkungen (Flüssigkeit, Feuer, elektrischer Strom...) schädigen die Haut und ggf. tiefere Gewebeschichten und führen $\mathrm{zu}$ einem teilweisen oder auch vollständigen Absterben des Gewebes. Die Schwe-
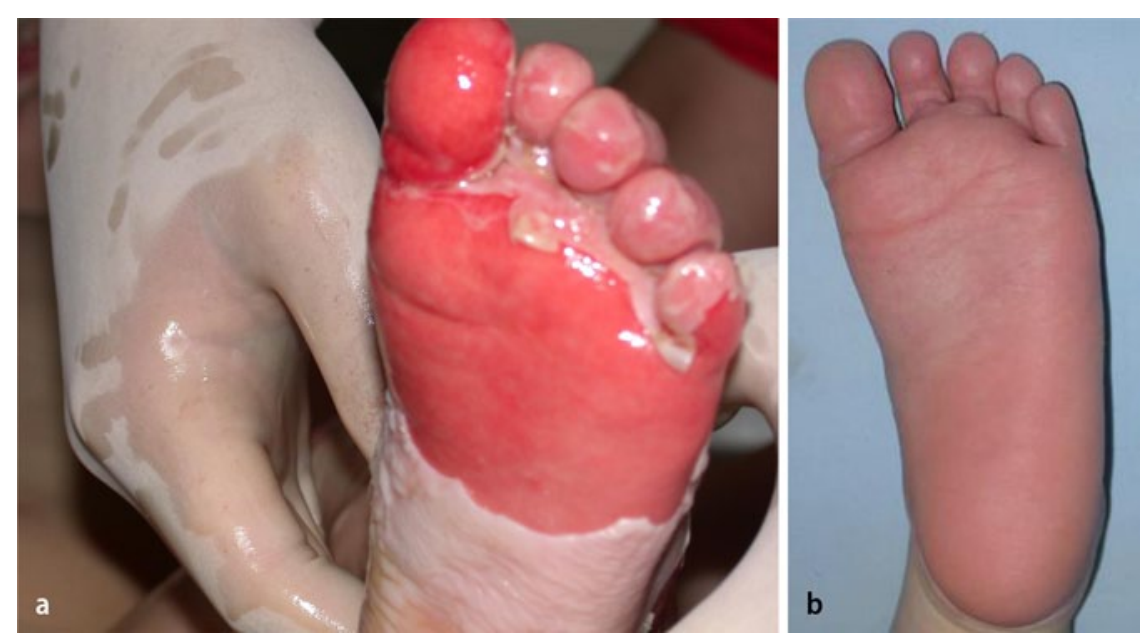

Abb. $1 \Delta$ Oberflächlich zweitgradige Brandverletzung: a nach Abtragen der Blasenbildung feuchter hyperämischer, deutlich schmerzempfindlicher Wundgrund mit prompter Rekapillarisierung auf Druck, b narbenfreies Ausheilungsergebnis nach 6 Wochen 


\section{Typische Frakturen und Luxationen}
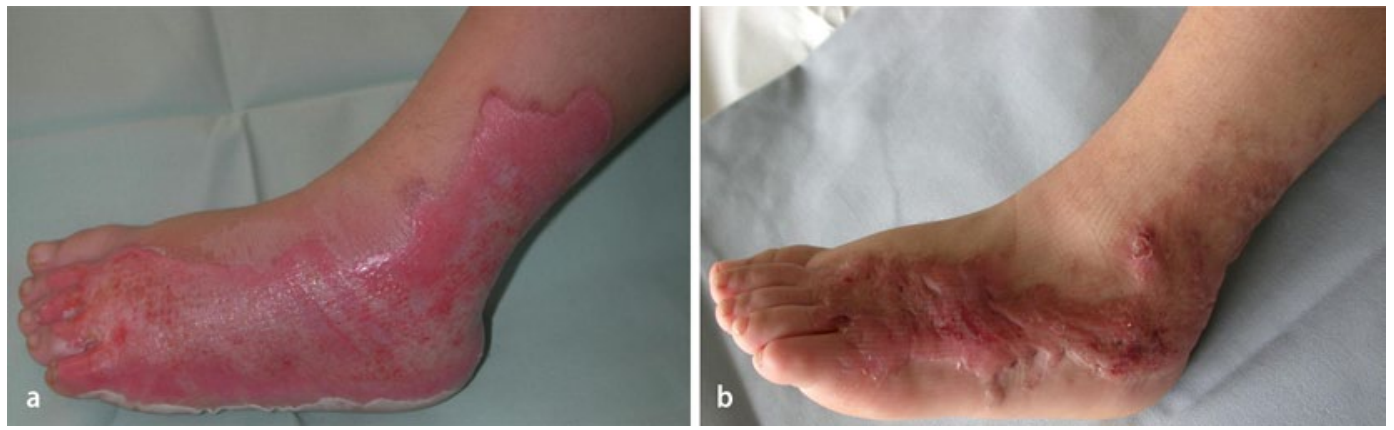

Abb. 2 \ Zentral tiefe zweitgradige Brandverletzung nach Verbrühung am Fußrücken: a nach Abtragung der Blasenreste grau-weißlicher, feuchter, kaum schmerzhafter Wundgrund mit deutlich verzögerter Rekapillarisierung auf Druck, b Ergebnis nach 6 Monaten und primär konservativer Therapie ohne tangentiales Débridement und ohne nachfolgende Spalthauttransplantation: ausgeprägt hypertrophe und instabile, stark juckende Narben mit beginnender Narbenkontraktur

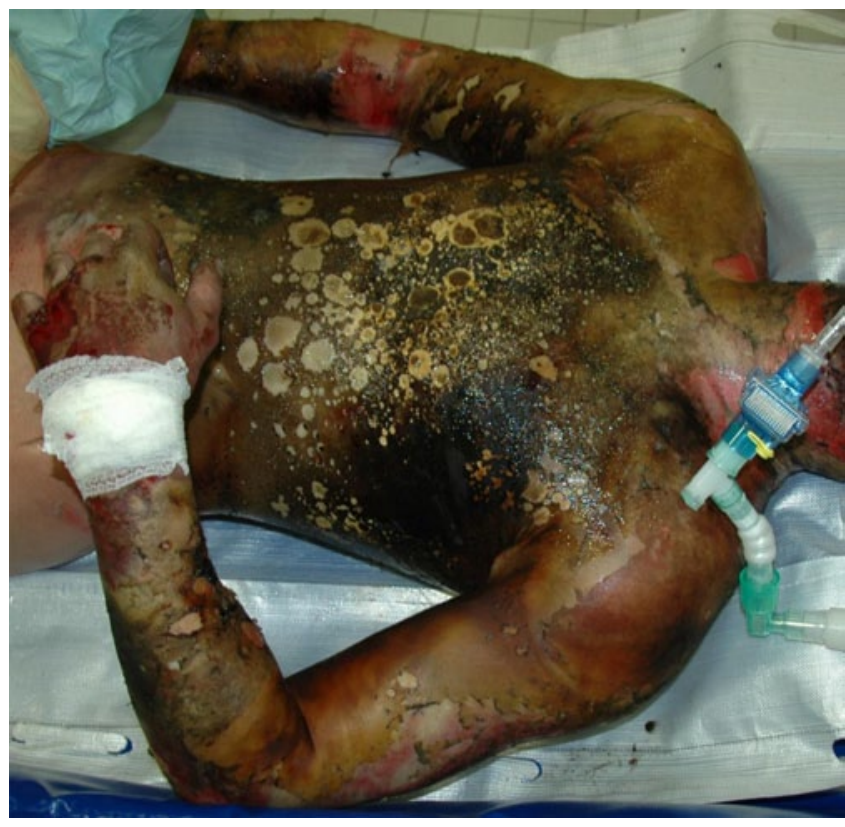

Abb. $3<$ Tiefe drittgradige zirkuläre Brandverletzung des Thorax und beider Arme mit vollschichtiger trockener Nekrose bis hin zur Verkohlung nach Lichtbogenverletzung beim S-BahnSurfen

Nicht nur, aber besonders nach Verbrühungen ist in den ersten Tagen nach der Verbrennung die Tiefenausdehnung des Gewebeschadens nicht immer sicher beurteilbar und wird dann zumeist auch unterschätzt. Häufig treten mehrere Stadien parallel mit fließenden Übergängen bei den jungen Patienten auf, sodass - auch abhängig von der Lokalisation der Verletzung - bei einem Patienten teils ein konservatives, teils ein operatives Vorgehen gewählt werden muss.

\section{Therapie}

Durch moderne Verfahren der konservativen Wundbehandlung, insbesondere der Wundunterdrucktherapie, sowie durch neueste Methoden der besonders präzi- sen, weil selektiven Entfernung von Nekrosen, beispielsweise dem $\mathrm{CO}_{2}$-Laser-Débridement oder dem enzymatischen Débridement ließ sich in den vergangenen Jahren am eigenen Patientengut der Unfallklinik Murnau die Notwendigkeit für Spalthautübertragungen um $1 / 3$ reduzieren.

Spalthauttransplantationspflichtige Verbrennungsareale sollten bei Kindern und Jugendlichen - so genügend Entnahmestellen zu Verfügung stehen - vorrangig mit ungemeshter Spalthaut gedeckt werden, um ein optimales, kosmetisches und funktionelles Ergebnis sicherstellen zu können. Insbesondere Gesichts-, Hals-, Dekolleté-, Hand- und Fußbereich profitieren davon. Wenn unumgänglich sollten ein Meshtransplantat möglichst eng- maschig oder aber auch die Meek-Technik bevorzugt werden.

Die Nachbehandlung von Kinderund Jugendlichen nach Brandverletzungen sollte im Rahmen interdisziplinärer Sprechstunden erfolgen. Ergo- und Physiotherapeuten, Orthopädietechniker und Chirurgen können dann gemeinsam die konservativen und chirurgisch-rekonstruktiv notwendigen Maßnahmen bis zum vollständigen Ausreifen der Narben und dem Abschluss des Wachstums der Patienten festlegen.

\section{Korrespondenzadresse}

\section{Dr. M.Öhlbauer}

Abteilung für Plastische Chirurgie und Verbrennungsmedizin, BG-Unfallklinik Murnau, Professor-Küntscher-Straße 8, 82418 Murnau Markus.Oehlbauer@bgu-murnau.de

\section{Einhaltung ethischer Richtlinien}

Interessenkonflikt. M. Öhlbauer gibt an, dass kein Interessenkonflikt besteht.

Dieser Beitrag beinhaltet keine Studien an Menschen oder Tieren.

The supplement containing this article is not sponsored by industry.

\section{Literatur}

1. AWMF (2011) Thermische und chemische Verletzungen. S1-Leitlinie der Deutschen Gesellschaft für Verbrennungsmedizin, Nr. 044/001. http:// www.awmf.org/uploads/tx_szleitlinien/044-001I_ S1_Thermische_und_Chemische_Verletzungen. pdf 
Tab. 1 Schema von Lund u. Browder zur Beurteilung der Ausdehnung von Brandverletzungen im Kindes- und Jugendalter (Angaben in Prozent Körperoberfläche)

\begin{tabular}{|c|c|c|c|c|c|c|c|}
\hline \multicolumn{2}{|c|}{ Betroffene Region } & $1 \mathrm{Jahr}$ & $\begin{array}{l}1 \text { bis } 4 \\
\text { Jahre }\end{array}$ & $\begin{array}{l}5 \text { bis } 9 \\
\text { Jahre }\end{array}$ & $\begin{array}{l}10 \text { bis } 14 \\
\text { Jahre }\end{array}$ & $\geq 15$ Jahre & Erwachsene \\
\hline \multicolumn{2}{|l|}{ Kopf } & 19 & 17 & 13 & 11 & 9 & 7 \\
\hline \multicolumn{2}{|l|}{ Hals } & 2 & 2 & 2 & 2 & 2 & 2 \\
\hline \multirow[t]{2}{*}{ Rumpf } & Vorne & 13 & 13 & 13 & 13 & 13 & 13 \\
\hline & Hinten & 13 & 13 & 13 & 13 & 13 & 13 \\
\hline \multirow[t]{2}{*}{ Gesäß } & Rechts & 2,5 & 2,5 & 2,5 & 2,5 & 2,5 & 2,5 \\
\hline & Links & 2,5 & 2,5 & 2,5 & 2,5 & 2,5 & 2,5 \\
\hline \multicolumn{2}{|l|}{ Genitalien } & 1 & 1 & 1 & 1 & 1 & 1 \\
\hline \multirow[t]{2}{*}{ Oberarm } & Rechts & 4 & 4 & 4 & 4 & 4 & 4 \\
\hline & Links & 4 & 4 & 4 & 4 & 4 & 4 \\
\hline \multirow[t]{2}{*}{ Unterarm } & Rechts & 3 & 3 & 3 & 3 & 3 & 3 \\
\hline & Links & 3 & 3 & 3 & 3 & 3 & 3 \\
\hline \multirow[t]{2}{*}{ Hand } & Rechts & 2,5 & 2,5 & 2,5 & 2,5 & 2,5 & 2,5 \\
\hline & Links & 2,5 & 2,5 & 2,5 & 2,5 & 2,5 & 2,5 \\
\hline \multirow[t]{2}{*}{ Oberschenkel } & Rechts & 5,5 & 6,5 & 8 & 8,5 & 9 & 9,5 \\
\hline & Links & 5,5 & 6,5 & 8 & 8,5 & 9 & 9,5 \\
\hline \multirow[t]{2}{*}{ Unterschenkel } & Rechts & 5 & 5 & 5,5 & 6 & 6,6 & 7 \\
\hline & Links & 5 & 5 & 5,5 & 6 & 6,6 & 7 \\
\hline \multirow[t]{2}{*}{ Fuß } & Rechts & 3,5 & 3,5 & 3,5 & 3,5 & 3,5 & 3,5 \\
\hline & Links & 3,5 & 3,5 & 3,5 & 3,5 & 3,5 & 3,5 \\
\hline
\end{tabular}

Trauma Berufskrankh 2015 - 17[Suppl 1]:241-243

DOI 10.1007/s10039-014-2126-y

๑) Springer-Verlag Berlin Heidelberg 2015

\section{M. Öhlbauer}

\section{Thermische Verletzungen im Kindes- und Jugendalter}

\section{Zusammenfassung}

Hintergrund. Ausgedehnte Brandverletzungen im Kindes- und Jugendalter zählen zu den schwersten Verletzungen des heranwachsenden Menschen. Vor allem Verbrühungs- und Stromverletzungen werden hinsichtlich ihrer Ausdehnung und Tiefe bei den jungen Patienten oft unterschätzt.

Ziel des Beitrags. Die diagnostische Problematik wird erläutert, und es werden Richtlinien für die korrekte Beurteilung gegeben sowie die Kriterien für die Überweisung in ein Brandverletztenzentrum dargestellt.

\section{Schlüsselwörter}

Kinder · Verbrennung · Verbrühung ·

Stromverletzung $\cdot$ Brandverletztenzentrum

\section{Thermal injuries in childhood and adolescence}

\section{Abstract}

Background. Extensive burn injuries in childhood and adolescence are one of the most severe forms of injury in growing people. The extent and depth of scalding and electric injuries in particular are often underestimated, especially in younger patients. Objectives. The diagnostic problems are discussed and guidelines for the correct assessment as well as the criteria for referral to a burn center are given.

\section{Keywords}

Children $\cdot$ Burns $\cdot$ Scalding $\cdot$ Electric injuries . Burn center 